\title{
Moderate versus light pressure massage therapy leads to greater weight gain in preterm infants
}

\author{
Tiffany Field ${ }^{\mathrm{a}, \mathrm{b}, *}$, Miguel A. Diego ${ }^{\mathrm{a}}$, Maria Hernandez-Reif ${ }^{\mathrm{a}}$, \\ Osvelia Deeds ${ }^{a}$, Barbara Figuereido ${ }^{c}$ \\ ${ }^{a}$ Touch Research Institutes, Department of Pediatrics, University of Miami School of Medicine, FL, United States \\ ${ }^{\mathrm{b}}$ Fielding Graduate University, CA, United States \\ c University of Minho, Portugal
}

Received 30 March 2006; received in revised form 31 May 2006; accepted 14 July 2006

\begin{abstract}
Sixty-eight preterm infants $(M \mathrm{GA}=30$ weeks) were randomly assigned to a moderate or to a light pressure massage therapy group to receive 15 massages three times per day for 5 days. Behavior state, stress behaviors and heart rate were recorded for 15 min before and during the first 15-min therapy session. Weight gain was recorded over the 5-day therapy period. The moderate versus light pressure massage group gained significantly more weight per day. During the behavior observations the moderate versus light pressure massage group showed significantly lower increases from the pre-session to the session recording on: (1) active sleep; (2) fussing; (3) crying; (4) movement; and (5) stress behavior (hiccupping). They also showed a smaller decrease in deep sleep, a greater decrease in heart rate and a greater increase in vagal tone. Thus, the moderate pressure massage therapy group appeared to be more relaxed and less aroused than the light pressure massage group which may have contributed to the greater weight gain of the moderate pressure massage therapy group.
\end{abstract}

(C) 2006 Elsevier Inc. All rights reserved.

Keywords: Massage therapy; Weight gain; Preterm infants

Moderate pressure massage therapy has been noted to be more effective than light pressure massage therapy for both adults and infants. In a study on adults, a greater decrease in heart rate and a positive shift toward left frontal FEG activation was noted for a moderate versus a light pressure massage therapy group (Diego, Field, Sanders, \& Hernandez-Reif, 2004). In addition, increased Delta and decreased Alpha and Beta activity indicated a relaxation response for the moderate pressure massage therapy group. The slow wave EEG activity (Delta power) is associated with lower arousal and relaxation (Niedermeyer \& Rodriguez, 1982) as are decreases in heart rate (Mok \& Wong, 2003). In contrast, the light pressure massage therapy group experienced increased heart rate and EEG pattern shifts indicative of increased arousal.

In a study on full-term infants receiving moderate versus light pressure massage therapy from their parents, the moderate pressure group showed greater gains in weight and length at the end of the first month of life (Field et al., 2004). They also showed greater improvements on the Brazelton Neonatal Behavior Assessment Scale following this daily massage study.

\footnotetext{
* Corresponding author at: Touch Research Institutes, University of Miami School of Medicine, P.O. Box 016820, Miami, FL 33101, United States. Tel.: +1 305243 6781; fax: +1 3052436488 .

E-mail address: tfield@med.miami.edu (T. Field).
} 
Finally, in a study comparing preterm infants receiving moderate pressure massage therapy versus those receiving light pressure massage, the moderate pressure group gained more weight and showed increased vagal activity and gastric motility during and after treatment (Diego, Field, \& Hernandez-Reif, 2005). Significant relations were noted between vagal activity, gastric motility and weight gain, but only for the moderate pressure massage therapy group. These relations were predicted in a model suggesting that stimulation of pressure receptors enhances vagal activity, which, in turn, stimulates gastric motility, which would then lead to more efficient food absorption and ultimately to greater weight gain (Field, 1988).

These data, combined, suggest that moderate pressure massage therapy may be more relaxing and less arousing physiologically. Stimulation of pressure receptors appears to contribute to this relaxed state, to decreased heart rate and to enhanced vagal activity, which, in turn, leads to the release of food absorption hormones such as insulin as measured in our laboratory (Field, 1988) and glucose and gastrin, as measured in the laboratory of UvnasMoberg (2004). This, in turn, would be expected to lead to greater weight gain, as in the model proposed by Field (1988).

The purpose of the present study was to observe the behavioral states and record heart rate and vagal activity in preterm infants receiving moderate versus those receiving light pressure massage therapy. Behavioral states have not been compared for moderate versus light massage therapy. The decreased heart rate in the adult study just described (Diego et al., 2004) and the increased vagal activity in the preterm infant study also just described (Diego et al., 2005) would suggest that more relaxed, less active states and lower arousal levels would be noted in the moderate pressure versus the light pressure massage therapy group. That is, less time in active aroused states, less stress behavior and lower heart rate would be expected for the moderate versus the light pressure group. In addition, as in the studies on full-term (Field et al., 2004) and preterm infants (Diego et al., 2004), greater weight gain was expected for the moderate versus light pressure massage therapy group. For this assessment preterm infants were randomly assigned to receive moderate pressure or light pressure massage therapy. Behavior state observations were made of sleep/wake states and stress behaviors, and heart rate was recorded before and during the therapy sessions, and weight gain was recorded across the 5-day massage therapy period.

\section{Method}

\subsection{Participants}

Sixty-eight preterm infants ( $M$ GA $=30$ weeks, $M$ BWT $=1292 \mathrm{~g}$ ) were randomly assigned to receive moderate or light pressure massage therapy three times per day for 5 days. This assignment was based on a stratification to ensure group similarity on factors noted to affect weight gain including gestational age, birth weight, days in the NICU ( $M=23$ days) and study entry weight $(M=1789 \mathrm{~g})$. The groups did not differ on these variables.

Preterm infants were recruited for this study if: (a) their gestational age (GA) was between 28 and 32 weeks; (b) their birth weight was between 550 and $1800 \mathrm{~g}$; (c) their NICU stay was 15-60 days; (d) their current weight at study entry was between 1000 and $1900 \mathrm{~g}$; (e) they were medically stable; (f) they were not receiving antibiotics or phototherapy. Preterm infants excluded from this study were those who: (a) exhibited genetic anomalies, congenital heart malformations and/or central nervous system dysfunction (e.g., intraventricular hemorrhage or a history of seizure); (b) were HIV positive; (c) had a history of maternal alcohol/illicit drug use (as determined by the mother's reported history, medical records and universal drug screen performed prior to delivery), syphilis or hepatitis B; or (d) required surgery. All participants continued to receive standard nursery care during the course of the study. Data collection was conducted by researchers blind to the group assignment. For 2 days prior, and throughout the 5-day study, relevant medical history was gathered, and weight gain was recorded.

\subsection{Procedures}

Treatment was provided for three 15-min periods per day, for 5 days approximately $1 \mathrm{~h}$ after feeding by massage therapists trained on the protocol. The therapists played no other role in the study, and they were blind to the hypotheses of the study. The therapists were randomly re-evaluated by the researchers to ensure protocol compliance especially with respect to the amount of pressure provided. The therapists providing the light pressure massage did not perform moderate massage therapy and vice versa. 
The moderate pressure massage therapy consisted of the 15-min preterm infant massage therapy protocol used by Field et al. (1986). The 15-min stimulation session consists of three standardized 5-min phases, tactile stimulation during the first and third phase and kinesthetic stimulation during the middle phase. During the tactile stimulation phase the infant is placed in a prone position and stroked with moderate pressure (e.g., slight skin color change from pink to white in a Caucasian baby or slight indentations in skin for all infants). The infants are massaged for five, 1-min periods (12 strokes at approximately $5 \mathrm{~s}$ per stroking motion) over each region in the following sequence: (1) from the top of the head to the neck and back to the top of the head; (2) from the neck across the shoulders to the neck; (3) from the upper back to the waist and back to the upper back; (4) from the thigh to the foot to the thigh both legs; and (5) from the shoulder to the hand to the shoulder on both arms. During the kinesthetic stimulation phase the infant is placed in a supine position and each arm, then each leg, and finally both legs together are flexed and extended (as in a bicycling motion). Each flexion/extension motion lasts $10 \mathrm{~s}$ for a total of five, 1-min segments.

The light pressure massage followed the same Field et al. (1986) protocol. The scheduling and duration of the light pressure massage sessions were identical to the moderate pressure sessions except that light pressure stroking was used during the first and last 5-min periods of the procedure. The middle 5-min period of kinesthetic stimulation remained the same.

\subsection{Measures}

\subsubsection{Sleep-wake behaviors}

Sleep-wake behaviors and behavior states were recorded on a laptop for the $15 \mathrm{~min}$ pre and $15 \mathrm{~min}$ during the massage. The behavior states were adapted from Thoman's sleep state criteria (Thoman, 1975) including quiet sleep, active sleep, REM sleep, drowsiness, inactive alert, active awake, fussing and crying. In addition, the following behaviors were recorded: hiccupping, clenching fists, yawning sneezing, startles, tremors and mouthing. The coders were trained to $90 \%$ reliability before the start of the study. Kappa coefficients for reliability testing on one third of the sample averaged .83 .

\subsection{2. $E K G$}

Electrocardiograms (EKG) were collected on the first day of the study. Physiological data were collected for a total of $30 \mathrm{~min}$ (15-min baseline and 15-min massage). Physiological data were collected at approximately the same time for each subject (between 1:00 p.m. and 3:00 p.m.) and exactly $1 \mathrm{~h}$ after feeding. Data were collected by researchers blind to the infant's group assignment. EKGs were acquired by placing three disposable silver chloride electrodes on the preterm infant's chest and back and amplifying and digitizing the signal using a UFI Model SRS2004/d-SP Electro-physiology Acquisition System with filters set at $1 \mathrm{~Hz}$ high pass and $100 \mathrm{~Hz}$ low pass and a gain of 2000. Data were sampled at a rate of 1000 samples per second and saved to a hard disk.

Following manual artifact correction, EKG data were converted to R-to-R-wave intervals (inter-beat interval) to the nearest millisecond using data acquisition and analysis software (Acq Knowledge software V.3.5, Biopac Systems Inc.). Inter-beat interval data were then analyzed using CMETI software (Allen, 2002) to obtain a measure of vagal tone (a.k.a. Respiratory Sinus Arrhythmia) (Allen, 2002; Porges, 1992).

\section{Results}

Repeated measures ANOVAS were conducted with pre-session and session values as the repeated measures, and Bonferroni $t$ tests were conducted on interaction effects. As can be seen in Table 1, the moderate versus the light pressure massage therapy group showed a greater increase in weight gain from the beginning to the end of the 5-day massage therapy period $(M=6 \mathrm{~g}$ versus $2 \mathrm{~g}$ increase per day) $(t=2.39, p<.02)$. On the behavioral observations, the moderate versus light pressure massage group showed: (1) a lower decrease in deep sleep (3\% time decrease versus $27 \%)(t=2.25, p<.05)$ from pre-session to session; (2) a lesser increase in active sleep (10\% versus $27 \%$ time $)(t=2.61$, $p<.02)$; (3) a lesser increase in fussing (.07\% versus $16 \%$ time) $(t=3.08, p<.01)$; (4) a lesser increase in crying $(.2 \%$ versus $10 \%$ time) $(t=2.72, p<.02)$; (5) a lesser increase in gross movement ( $4 \%$ versus $8 \%$ time $)(t=2.28, p<.05)$; and (6) a lesser increase in stress behavior (hiccupping) ( $1 \%$ versus $5 \%$ time) $(t=3.40, p<.01)$. In addition, the moderate pressure massage therapy group showed a greater decrease in heart rate (6BPM decrease versus 0 BPM decrease) $(t=3.64, p<.01)$ which translated into a greater increase in vagal tone (.3 versus 0 increase $)(t=2.3, p<.05)$. 
Table 1

Means for neonatal measures for moderate and light pressure massage therapy groups pre and during therapy

\begin{tabular}{|c|c|c|c|c|}
\hline \multirow[t]{3}{*}{ Measure } & \multicolumn{4}{|c|}{ Groups } \\
\hline & \multicolumn{2}{|c|}{ Moderate } & \multicolumn{2}{|l|}{ Light } \\
\hline & Pre & During & Pre & During \\
\hline Weight gain (g) & 22.2 & $27.8^{\mathrm{a}}$ & 28.8 & 27.4 \\
\hline Deep sleep (\% time) & 38.9 & 35.9 & 34.5 & $7.9^{\mathrm{a}}$ \\
\hline Fussing ( $\%$ time) & 0.1 & 0.2 & 0.0 & $16.0^{\mathrm{a}}$ \\
\hline Crying (\% time) & 0.6 & 0.7 & 1.8 & $11.6^{\mathrm{a}}$ \\
\hline Movement (\% time) & 6.9 & 6.1 & 9.9 & $18.0^{\mathrm{a}}$ \\
\hline Hiccuping (\% time) & 1.6 & 2.6 & 2.9 & $7.7^{\mathrm{b}}$ \\
\hline Heart rate (BPM) & 171.1 & $164.6^{\mathrm{a}}$ & 168.0 & 168.7 \\
\hline
\end{tabular}

${ }^{\mathrm{a}} p<.05$.

${ }^{\mathrm{b}} p<.01$.

\section{Discussion}

The moderate pressure massage therapy group gained more weight than the light pressure massage group. The increased weight gain shown by the moderate pressure massage group is consistent with increased weight gain in many studies on both preterm infants (Diego et al., 2004; Dieter, Field, Hernandez-Reif, Emory, \& Redzepi, 2003; Field et al., 1986) and full-term infants (Field et al., 2004; Goldstein-Ferber, 2004; Moyer-Mileur, Brunstetter, McNaught, Gill, \& Chan, 2000).

These data also suggest that the moderate pressure massage therapy group was more relaxed and less aroused than the light pressure massage therapy group. This was evident in their showing less stressed and more relaxed behavior states (more deep sleep and less active sleep, fussing, crying, less movement and less hiccupping) and lower heart rate. These findings are consistent with the greater relaxation and lesser arousal noted in adults receiving moderate pressure massage therapy who showed lower heart rate and more relaxed EEG patterns (Diego et al., 2004) and the full-term infants who showed better growth and performance on the Brazelton scale after one month of moderate pressure massage (Field et al., 2004). The decreased heart rate and increased vagal tone are also consistent with the data on massaged preterm infants presented by Diego et al. (2004).

Being in a more relaxed, less active, less aroused state during the moderate pressure massage therapy sessions may have contributed to the greater weight gain, potentially via less energy expenditure. Future studies would need to conduct additional behavior observations across the study period. In addition, energy expenditure could be measured, for example, by oxygen consumption and metabolic activity to determine how they are related to weight gain. In the interim, these data at least suggest the importance of providing moderate pressure during massage therapy with preterm infants.

\section{Acknowledgments}

We would like to thank the mothers and infants who participated in this study and Julia Beutler, Larissa Feijo, Karla Gill and Yanexy Vera for their help with participant recruitment and data collection. We would also like to thank John Allen for his help with EKG analysis software. This research was supported by Senior Research Scientist Awards (\#MH00331 and \#AT01585) and an NIHM merit award (MH \#46586) and a March of Dimes Grant (\#12-FY03-48) to Tiffany Field, an NCCAM research Grant to Maria Hernandez-Reif (\#AT00370) and an NCCAM research supplement (\#AT00370-02S 1) to Miguel A. Diego and funding from Johnson and Johnson Pediatric Institute.

\section{References}

Allen, J. J. B. (2002). Calculating metrics of cardiac chronotropy: A pragmatic overview. Psychophysics, 39, S18.

Diego, M., Field, T., \& Hernandez-Reif, M. (2005). Vagal activity, gastric motility, and weight gain in massaged preterm neonates. The Journal of Pediatrics, 147, 50-55. 
Diego, M., Field, T., Sanders, C., \& Hernandez-Reif, M. (2004). Massage therapy of moderate and light pressure and vibrator effects on EEG and heart rate. International Journal of Neuroscience, 114, 31-45.

Dieter, J. N., Field, T., Hernandez-Reif, M., Emory, E., \& Redzepi, M. (2003). Stable preterm infants gain more weight and sleep less following 5 days of massage therapy. Journal of Pediatric Psychology, 28(6), 403-411.

Field, T. (1988). Stimulation of preterm infants. Pediatrics Review, 10, 149-154.

Field, T., Hernandez-Reif, M., Diego, M., Feijo, L., Vera, Y., \& Gil, K. (2004). Massage therapy by parents improves early growth and development. Infant Behavior and Development, 27(4), 435-442.

Field, T., Schanberg, S., Scafidi, F., Bauer, C. R., Vega-Lahr, N., Garcia, R., et al. (1986). Tactile/kinesthetic stimulation effects on preterm neonates. Pediatrics, 77(5), 654-658.

Goldstein-Ferber, S. (2004). Massage therapy and sleep-wake rhythms in the neonate. In T. Field (Ed.), Touch and massage in early child development. Johnson \& Johnson Pediatric Institute, L.L.C.

Mok, E., \& Wong, K. Y. (2003). Effects of music on patient anxiety. AORN, 77, 396-397.

Moyer-Mileur, L. J., Brunstetter, V., McNaught, T. P., Gill, G., \& Chan, G. M. (2000). Daily physical activity program increases bone mineralization and growth in preterm very low birth weight infants. Pediatrics, 106, 1088-1092.

Niedermeyer, E., \& Rodriguez, I. (1982). The aphasia-epilepsy syndrome in children: Electroencephalographic aspect. Clinical Electroencephalography, 13(1), 23-35.

Porges, S. W. (1992). Vagal tone: A physiologic marker of stress vulnerability. Pediatrics, 90(3 Pt 2), 498-504.

Thoman, E. B. (1975). Early development of sleeping behaviors in infants. In N. T. Ellis (Ed.), Behavior and development in infancy: Human and animal studies. New York: John Wiley and Sons.

Uvnas-Moberg, K. (2004). Massage relaxation and well-being: A possible role for oxytocin as an integrative principle. In T. Field (Ed.), Touch and massage in early child development. Johnson \& Johnson Pediatric Institute, L.L.C. 